\title{
Synchronization of Complex Networks with Random Coupling Strengths and Mixed Probabilistic Time-Varying Coupling Delays Using Sampled Data
}

\author{
Jian-An Wang \\ School of Electronics Information Engineering, Taiyuan University of Science and Technology, Taiyuan, Shanxi 030024, China \\ Correspondence should be addressed to Jian-An Wang; wangjianan588@163.com
}

Received 18 November 2013; Revised 27 April 2014; Accepted 28 April 2014; Published 19 May 2014

Academic Editor: Allan Peterson

Copyright (C) 2014 Jian-An Wang. This is an open access article distributed under the Creative Commons Attribution License, which permits unrestricted use, distribution, and reproduction in any medium, provided the original work is properly cited.

\begin{abstract}
The sampled-data synchronization problem for complex networks with random coupling strengths, probabilistic time-varying coupling delay, and distributed delay (mixed delays) is investigated. The sampling period is assumed to be time varying and bounded. By using the properties of random variables and input delay approach, new synchronization error dynamics are constructed. Based on the delay decomposition method and reciprocally convex approach, a delay-dependent mean square synchronization condition is established in terms of linear matrix inequalities (LMIs). According to the proposed condition, an explicit expression for a set of desired sampled-data controllers can be achieved by solving LMIs. Numerical examples are given to demonstrate the effectiveness of the theoretical results.
\end{abstract}

\section{Introduction}

It is well known that many large-scale systems, such as the Internet, World Wide Web, metabolic pathways, food webs, electric power grids, and social networks, can be modeled by complex networks [1,2]. A real-world network often consists of a large number of interconnected nodes, in which each node represents an element with certain dynamical system and edge represents the interactions among them. Synchronization, as a typical collective behavior, is an important topic in complex networks. In the past few decades, much effort has been devoted to the study of synchronization in complex networks [3-12]. The main reason for this is that network synchronization can not only explain many natural phenomena but also has many potential applications, such as secure communication, synchronous information exchange over the Internet, and the synchronous transfer of digital signals in communication networks.

With the rapid development of modern microprocessor, sampled-data control system has been investigated extensively by various researchers [13-16]. In the sampled-data control system, the control signals are allowed to change only at discrete sampling instants, which can drastically reduce the amount of information transmitted and increase the efficiency of bandwidth usage. The input delay approach [13], which is based on modeling the sampled-data system as a continuous-time system with a time-varying sawtooth delay in the control input, is an important approach in the study of sampled control system. Furthermore, by constructing a time-dependent Lyapunov functional, a refined input delay approach [14] was proposed. Based on the input delay and refined input delay approach, the sampled-data synchronization problems in Lure system or delayed neural networks were studied [17-19]. Recently, in the framework of the input delay approach, the authors in [20] investigated the sampleddata synchronization problem for a class of general complex networks with time-varying coupling delays. By considering the neglected terms in the derivative of Lyapunov functional in [20], some improved sampled-data synchronization criterion was derived in [21], and the desired sampled-data feedback controllers were designed in terms of the solution to certain linear matrix inequalities (LMIs). In [22], by using the refined input delay approach and convex combination technique, the sampled-data exponential synchronization for 
complex dynamical networks with time-varying coupling delay and uncertain sampling has been considered.

Time delay is ubiquitous in many physical systems. In order to give a more precise description of dynamical network, time delay should be given more attention inevitably. Therefore, the synchronization for a complex network with coupling delays has been investigated by many researchers. In general, coupling delay of complex networks is deterministic. However, as illustrated in [23], coupling delay often exists in a random form; that is to say, some values of the delay are very large, but the probability of the delay taking such large values is very small. This may lead to a more conservative result if only the information of variation range of the delay is considered. It is worth mentioning that time delays can be generally categorized as discrete ones and distributed ones. Moreover, it has been observed that they usually have a spatial nature due to the presence of a number of parallel pathways of a variety of axon sizes and lengths in a network. Therefore, it is interesting and meaningful to investigate the synchronization in a dynamical network with probabilistic time-varying coupling delay as well as distributed delay (mixed delays).

On the other hand, because of the effects of environment and artificial factor, the coupling strength of complex network may randomly vary around some constants [23, 24]. Thus, random phenomena in coupling strength should be taken into account when dealing with the synchronization of complex networks. If only the upper or lower bound of the random coupling strength is considered, some conservative result will be derived. Furthermore, the normal distribution characteristic of random variables can be easily obtained by statistical methods. Therefore, it is interesting to investigate the synchronization of complex networks with random coupling strength described by normal distribution. To the best of our knowledge, the synchronization problem of complex networks with mixed coupling delays and random coupling strengths based on sampled-data control has not been studied in the literature. This is the motivation of our paper.

Motivated by the aforementioned discussion, in this paper, the problem of sampled-data synchronization is investigated for complex network with random coupling strengths and mixed probabilistic time-varying coupling delays. The sampling period is assumed to be time varying but bounded by a known positive constant. On the basis of the properties of random variables and the input delay approach, new error dynamics is obtained. By using the delay decomposition method and reciprocally convex approach, a mean square synchronization condition is derived in terms of LMIs. Based on the proposed condition, the corresponding desired sampled-data controllers are designed, which can be solved effectively by using MATLAB LMI Toolbox. Finally, numerical examples are given to illustrate the effectiveness of the obtained results.

Notations. Throughout this paper, $R^{n}$ and $R^{m \times n}$ denote the $n$-dimensional Euclidean space and the set of all $m \times n$ real matrices, respectively. $P>0$ or $P<0$ means that $P$ is symmetric and positive or negative definite. The superscript " $T$ " represents the transpose, and " $I$ " and " 0 " denote the identity and zero matrices with appropriate dimensions. $\operatorname{diag}\left\{l_{1}, l_{2}, \ldots, l_{n}\right\}$ stands for a block diagonal matrix. $\|\cdot\|$ denotes the Euclidean norm of a vector. The symmetric terms in a symmetric matrix are denoted by $* . \lambda_{\min }(A)$ is the minimum eigenvalue of symmetric matrix $A$. The notation $A \otimes B$ represents the Kronecker product of matrices $A$ and $B$.

\section{Preliminaries and Model Description}

Consider a hybrid coupled complex network consisting of $N$ identical coupled nodes as follows:

$$
\begin{aligned}
\dot{x}_{i}(t)= & f\left(x_{i}(t)\right)+c_{1}(t) \sum_{j=1}^{N} G_{i j} D x_{j}(t) \\
& +c_{2}(t) \sum_{j=1}^{N} G_{i j} A x_{j}(t-\tau(t)) \\
& +c_{3}(t) \sum_{j=1}^{N} G_{i j} B \int_{t-r(t)}^{t} x_{j}(s) d s+u_{i}(t), \\
& \quad i=1,2, \ldots, N
\end{aligned}
$$

where $x_{i}=\left(x_{i 1}, x_{i 2}, \ldots, x_{i n}\right) \in R^{n}$ and $u_{i}(t) \in R^{n}$ are, respectively, the state variable and the control input of the node $i . f: R^{n} \rightarrow R^{n}$ is a continuous vector-valued function. $\tau(t) \in\left[0, h_{2}\right]$ and $r(t) \in[0, r]$ denote the time-varying coupling delay and distributed coupling delay, respectively. $c_{k}(t), k=1,2,3$ are mutually independent random variables, which denote the coupling strengths of no-delayed coupling, time-delayed coupling, and distributed coupling, respectively. $D, A, B \in R^{n \times n}$ are the inner-coupling matrices. The coupling configuration matrix $G=\left(G_{i j}\right)_{N \times N}$ is defined as follows: if there is a connection between node $i$ and node $j(i \neq j)$, then $G_{i j}=G_{j i}=1$; otherwise, $G_{i j}=G_{j i}=0$, and the diagonal elements of matrix $G$ are defined by $G_{i i}=$ $-\sum_{j=1, j \neq i}^{N} G_{i j}=-\sum_{j=1, j \neq i}^{N} G_{j i}, i=1,2, \ldots, N$.

Throughout this paper, the following assumptions are made.

Assumption 1. The time-varying coupling delay $\tau(t)$ satisfies $0 \leq h_{1} \leq \tau(t) \leq h_{2}$. Furthermore, the probability distribution of $\tau(t)$ taking values in $\left[0, h_{1}\right)$ and $\left[h_{1}, h_{2}\right]$ is known a priori.

Under Assumption 1, the probability distribution of $\tau(t)$ is assumed to be $\operatorname{Prob}\left\{\tau(t) \in\left[0, h_{1}\right)\right\}=\beta_{0}$, and $\operatorname{Prob}\{\tau(t) \in$ $\left.\left[h_{1}, h_{2}\right]\right\}=1-\beta_{0}$, where $0 \leq \beta_{0} \leq 1$ is a constant. Therefore, a stochastic Bernoulli variable can be defined as

$$
\beta(t)= \begin{cases}1, & \tau(t) \in\left[0, h_{1}\right), \\ 0, & \tau(t) \in\left[h_{1}, h_{2}\right] .\end{cases}
$$

It can be derived from (2) that

$$
\begin{gathered}
\operatorname{Prob}\{\beta(t)=1\}=E\{\beta(t)\}=\beta_{0}, \\
\operatorname{Prob}\{\beta(t)=0\}=1-E\{\beta(t)\}=1-\beta_{0} .
\end{gathered}
$$


Furthermore, from the definition of $\beta(t)$, it is easy to check that

$$
\begin{gathered}
E\left\{\beta_{0}-\beta(t)\right\}=0, \\
E\left\{\left(\beta_{0}-\beta(t)\right)^{2}\right\}=\beta_{0}\left(1-\beta_{0}\right) .
\end{gathered}
$$

Now, we introduce two time-varying delays $\tau_{1}(t)$ and $\tau_{2}(t)$ such that

$$
\tau(t)= \begin{cases}\tau_{1}(t), & \tau(t) \in\left[0, h_{1}\right), \\ \tau_{2}(t), & \tau(t) \in\left[h_{1}, h_{2}\right] .\end{cases}
$$

Assumption 2. There exist constants $\mu_{1}$ and $\mu_{2}$ such that $\dot{\tau}_{1}(t) \leq \mu_{1}<\infty$ and $\dot{\tau}_{2}(t) \leq \mu_{2}<\infty$.

By using the new functions $\tau_{1}(t), \tau_{2}(t)$, and $\beta(t)$, system (1) can be rewritten as

$$
\begin{aligned}
\dot{x}_{i}= & f\left(x_{i}(t)\right)+c_{1}(t) \sum_{j=1}^{N} G_{i j} D x_{j}(t) \\
& +\beta(t) c_{2}(t) \sum_{j=1}^{N} G_{i j} A x_{j}\left(t-\tau_{1}(t)\right) \\
& +(1-\beta(t)) c_{2}(t) \sum_{j=1}^{N} G_{i j} A x_{j}\left(t-\tau_{2}(t)\right) \\
& +c_{3}(t) \sum_{j=1}^{N} G_{i j} B \int_{t-r(t)}^{t} x_{j}(s) d s+u_{i}(t), \\
& i=1,2, \ldots, N .
\end{aligned}
$$

For the random coupling strengths $c_{k}(t), k=1,2,3$, similar to $[23,24]$, we assume that almost all the values of them are taken on some nonnegative intervals; that is, $c_{k}(t) \epsilon$ $\left(\sigma_{k}, \rho_{k}\right)$, where $\sigma_{k}$ and $\rho_{k}$ are nonnegative constants. It should be pointed out that almost all the values of $c_{k}(t)$ satisfying $c_{k}(t) \in\left(\sigma_{k}, \rho_{k}\right)$ just imply that $\operatorname{Prob}\left\{c_{k}(t) \in\left(\sigma_{k}, \rho_{k}\right)\right\}=1$, $\operatorname{Prob}\left\{c_{k}(t)<\sigma_{k}\right\}=0$, and $\operatorname{Prob}\left\{c_{k}(t)>\rho_{k}\right\}=0$. It does not mean that the minimum and maximum allowable coupling strength bounds of $c_{k}(t)$ are $\sigma_{k}$ and $\rho_{k}$, respectively. Thus, the actual lower bound and upper bound of $c_{k}(t)$ may be very small and be very large, respectively. Clearly, it is very different from the constant coupling strength.

Assumption 3. The mathematical exception and variance of $c_{k}(t)$ are $E\left\{c_{k}(t)\right\}=c_{k 0}$ and $E\left\{\left(c_{k}(t)-c_{k 0}\right)^{2}\right\}=\delta_{k}^{2}$, respectively, where $c_{k 0}$ and $\delta_{k}$ are nonnegative constants.

Based on the property of variables $c_{k}(t)$, system (6) can be further expressed by

$$
\begin{aligned}
\dot{x}_{i}= & f\left(x_{i}(t)\right)+c_{10} \sum_{j=1}^{N} G_{i j} D x_{j}(t) \\
& +\left(c_{1}(t)-c_{10}\right) \sum_{j=1}^{N} G_{i j} D x_{j}(t)
\end{aligned}
$$

$$
\begin{aligned}
& +\beta(t) c_{20} \sum_{j=1}^{N} G_{i j} A x_{j}\left(t-\tau_{1}(t)\right) \\
& +\beta(t)\left(c_{2}(t)-c_{20}\right) \sum_{j=1}^{N} G_{i j} A x_{j}\left(t-\tau_{1}(t)\right) \\
& +(1-\beta(t)) c_{20} \sum_{j=1}^{N} G_{i j} A x_{j}\left(t-\tau_{2}(t)\right) \\
& +(1-\beta(t))\left(c_{2}(t)-c_{20}\right) \sum_{j=1}^{N} G_{i j} A x_{j}\left(t-\tau_{2}(t)\right) \\
& +c_{30} \sum_{j=1}^{N} G_{i j} B \int_{t-r(t)}^{t} x_{j}(s) d s \\
& +\left(c_{3}(t)-c_{30}\right) \sum_{j=1}^{N} G_{i j} B \int_{t-r(t)}^{t} x_{j}(s) d s \\
& +u_{i}(t), \quad i=1,2, \ldots, N .
\end{aligned}
$$

Remark 4. The network model considered here is more general. It is worth noting that when $\beta(t)=1, c_{1}(t)=c_{10}$, $c_{2}(t)=c_{20}$, and the distributed coupling is missing, system (7) includes the models considered in $[20,21]$ as a special case.

Assumption 5. The nonlinear continuous vector-valued function $f$ is assumed to satisfy the following sector-bounded condition:

$$
[f(x)-f(y)-U(x-y)]^{T}[f(x)-f(y)-V(x-y)] \leq 0
$$

for all $x, y \in R^{n}$, where $U$ and $V$ are constant matrices of appropriate dimensions.

Let $e_{i}(t)=x_{i}(t)-s(t)$ be the synchronization error, where $s(t) \in R^{n}$ is the state trajectory of the unforced isolate node $\dot{s}(t)=f(x(t))$. Then, the error dynamics can be obtained as follows:

$$
\begin{aligned}
\dot{e}_{i}= & g\left(e_{i}(t)\right)+c_{10} \sum_{j=1}^{N} G_{i j} D e_{j}(t) \\
& +\left(c_{1}(t)-c_{10}\right) \sum_{j=1}^{N} G_{i j} D e_{j}(t) \\
& +\beta(t) c_{20} \sum_{j=1}^{N} G_{i j} A e_{j}\left(t-\tau_{1}(t)\right) \\
& +\beta(t)\left(c_{2}(t)-c_{20}\right) \sum_{j=1}^{N} G_{i j} A e_{j}\left(t-\tau_{1}(t)\right)
\end{aligned}
$$




$$
\begin{aligned}
& +(1-\beta(t)) c_{20} \sum_{j=1}^{N} G_{i j} A e_{j}\left(t-\tau_{2}(t)\right) \\
& +(1-\beta(t))\left(c_{2}(t)-c_{20}\right) \sum_{j=1}^{N} G_{i j} A e_{j}\left(t-\tau_{2}(t)\right) \\
& +c_{30} \sum_{j=1}^{N} G_{i j} B \int_{t-r(t)}^{t} e_{j}(s) d s \\
& +\left(c_{3}(t)-c_{30}\right) \sum_{j=1}^{N} G_{i j} B \int_{t-r(t)}^{t} e_{j}(s) d s \\
& +u_{i}(t), \quad i=1,2, \ldots, N,
\end{aligned}
$$

where $g\left(e_{i}(t)\right)=\left[\begin{array}{llll}f_{1}\left(e_{i 1}(t)\right) & f_{2}\left(e_{i 2}(t)\right) & \cdots & f_{n}\left(e_{i n}(t)\right)\end{array}\right]^{T}$, $f_{j}\left(e_{i j}(t)\right)=f_{j}\left(x_{i j}(t)\right)-f_{j}\left(s_{j}(t)\right)$.

The control signal is assumed to be generalized by using a zero-order hold $(\mathrm{ZOH})$ function with a sequence of hold times $0=t_{0}<t_{1}<\cdots<t_{k}<\cdots$. Therefore, the state feedback controller takes the following form:

$$
u_{i}=K_{i} e_{i}\left(t_{k}\right), \quad t_{k} \leq t<t_{k+1}, \quad i=1,2, \ldots, N,
$$

where $K_{i}$ is the feedback gain matrix to be designed, $e_{i}\left(t_{k}\right)$ is the discrete measurement of $e_{i}(t)$ at sampling instant $t_{k}$, and $\lim _{k \rightarrow+\infty} t_{k}=+\infty$. In this paper, the sampling is not required to be periodic, and the only assumption is that the distance between any two consecutive sampling instants is less than a given bound. It is assumed that $t_{k+1}-t_{k}=h_{k} \leq p$ for any integer $k \geq 0$, where $p>0$ represents the largest sampling interval.

By using the input delay approach and the Kronecker product, error dynamics (9) can be rewritten in the following compact form:

$$
\begin{aligned}
\dot{e}(t)= & \bar{g}(e(t))+c_{10}(G \otimes D) e(t) \\
& +\left(c_{1}(t)-c_{10}\right)(G \otimes D) e(t) \\
& +\beta(t) c_{20}(G \otimes A) e\left(t-\tau_{1}(t)\right) \\
& +\beta(t)\left(c_{2}(t)-c_{20}\right)(G \otimes A) e\left(t-\tau_{1}(t)\right) \\
& +(1-\beta(t)) c_{20}(G \otimes A) e\left(t-\tau_{2}(t)\right) \\
& +(1-\beta(t))\left(c_{2}(t)-c_{20}\right)(G \otimes A) e\left(t-\tau_{2}(t)\right) \\
& +c_{30}(G \otimes B) \int_{t-r(t)}^{t} e(s) d s \\
& +\left(c_{3}(t)-c_{30}\right)(G \otimes B) \int_{t-r(t)}^{t} e(s) d s \\
& +K e(t-d(t)),
\end{aligned}
$$

where $e(t)=\operatorname{col}\left\{e_{1}(t), e_{2}(t), \ldots, e_{N}(t)\right\}, \bar{g}(e(t))=$ $\left[g^{T}\left(e_{1}(t)\right) g^{T}\left(e_{2}(t)\right) \cdots g^{T}\left(e_{n}(t)\right)\right]^{T}, K=\operatorname{diag}\left\{K_{1}, K_{2}, \ldots\right.$, $\left.K_{N}\right\}, d(t)=t-t_{k}$.
The purpose of this paper is focusing on designing a set of sampled-data controllers (10) with sampling period as big as possible to ensure synchronizing the delayed complex network (6) in mean square sense. Obviously, the synchronization problem has been converted into the mean square asymptotical stability problem of error system (11). Therefore, there are two main issues to be considered in this paper: one is to find some sufficient conditions for the error system (11) to be globally stable in mean square for given $K_{i}$, and the other is to derive the design method of sampleddata controllers. To obtain the main results, the following definition and lemmas will be needed.

Definition 6. The coupled complex network (6) is said to be globally synchronized in mean square sense if $\lim _{t \rightarrow \infty} E\left\{\left\|e_{i}(t)\right\|^{2}\right\}=0, i=1,2, \ldots, N$ holds for any initial values.

Lemma 7 (see [25] (Jensen's inequality)). For any positive symmetric constant matrix $Z=Z^{T}>0$, scalar $h_{1}<h_{2}$ and vector function $\omega:\left[h_{1}, h_{2}\right] \rightarrow R^{n}$ such that the integrations concerned are well defined; then

$$
\begin{gathered}
-\left(h_{2}-h_{1}\right) \int_{t-h_{2}}^{t-h_{1}} \omega^{T}(s) Z \omega(s) d s \\
\leq-\int_{t-h_{2}}^{t-h_{1}} \omega^{T}(s) d s Z \int_{t-h_{2}}^{t-h_{1}} \omega(s) d s .
\end{gathered}
$$

Lemma 8 (see [26] (reciprocally convex approach)). Let $f_{1}, f_{2}, \ldots, f_{N}: R^{m} \mapsto R$ have positive values in an open subset $D$ of $R^{m}$. Then, the reciprocally convex combination of $f_{i}$ over $D$ satisfies

$$
\min _{\left\{\alpha_{i} \mid \alpha_{i}>0, \sum_{i} \alpha_{i}=1\right\}} \sum_{i} f_{i}(t)=\sum_{i} f_{i}(t)+\max _{g_{i, j}(t)} \sum_{i \neq j} g_{i, j}(t)
$$

subject to

$$
\left\{g_{i, j}: R^{m} \longmapsto R, g_{j, i}(t) \triangleq g_{i, j}(t),\left[\begin{array}{cc}
f_{i}(t) & g_{i, j}(t) \\
g_{j, i}(t) & f_{j}(t)
\end{array}\right] \geq 0\right\} .
$$

\section{Main Results}

In this section, we will first give a sufficient condition, which can guarantee the error system (11) to be globally stable in mean square sense. Then, based on the given synchronization condition, we propose an explicit expression of the sampleddata controllers. Before presenting the main results, for the sake of presentation simplicity, we denote

$$
\begin{gathered}
\bar{U}=\frac{\left(I_{N} \otimes U\right)^{T}\left(I_{N} \otimes V\right)}{2}+\frac{\left(I_{N} \otimes V\right)^{T}\left(I_{N} \otimes U\right)}{2} \\
\bar{V}=-\frac{\left(I_{N} \otimes U\right)^{T}+\left(I_{N} \otimes V\right)^{T}}{2} .
\end{gathered}
$$


Theorem 9. Suppose that Assumptions 1, 2, 3, and 5 hold. For given controller gain matrices $K_{i}$, the error system (11) is globally asymptotically stable in mean square sense if there exist matrices $P>0,\left[\begin{array}{cc}\mathrm{Q}_{1} & \mathrm{Q}_{2} \\ * & \mathrm{Q}_{3}\end{array}\right]>0, Q_{i}>0(i=4, \ldots, 7), Z_{i}>$ $0(i=1, \ldots, 5), S_{i}(i=1, \ldots, 4)$ of appropriate dimensions and a scalar $\varepsilon>0$ such that the following LMIs hold:

$$
\begin{gathered}
{\left[\begin{array}{cc}
Z_{k} & S_{k} \\
* & Z_{k}
\end{array}\right] \geq 0, \quad k=1, \ldots, 4,} \\
{\left[\begin{array}{cc}
\Phi_{i} & \Gamma \\
* & \Psi
\end{array}\right]<0, \quad i=1,2,}
\end{gathered}
$$

where

$$
\begin{aligned}
& \Phi_{1}=\Sigma-\Delta_{1}^{T} Z_{1} \Delta_{1}-\Delta_{2}^{T} Z_{1} \Delta_{2}-\Delta_{1}^{T} S_{1} \Delta_{2}-\Delta_{2}^{T} S_{1}^{t} \Delta_{1}-\Delta_{5}^{T} S_{3} \Delta_{6}-\Delta_{6}^{T} S_{3}^{T} \Delta_{5} \\
& -\Delta_{7}^{T} S_{4} \Delta_{8}-\Delta_{8}^{T} S_{4}^{T} \Delta_{7}-\Delta_{9}^{T} Z_{2} \Delta_{9} \\
& \Phi_{2}=\Sigma-\Delta_{3}^{T} Z_{2} \Delta_{3}-\Delta_{4}^{T} Z_{2} \Delta_{4}-\Delta_{3}^{T} S_{2} \Delta_{4}-\Delta_{4}^{T} S_{2}^{T} \Delta_{3}-\Delta_{5}^{T} S_{3} \Delta_{6}-\Delta_{6}^{T} S_{3}^{T} \Delta_{5} \\
& -\Delta_{7}^{T} S_{4} \Delta_{8}-\Delta_{8}^{T} S_{3}^{T} \Delta_{7}-\Delta_{10}^{T} Z_{1} \Delta_{10} \\
& \Sigma=\left[\begin{array}{cccccccccc}
\Sigma_{11} & P K & Q_{2} & 0 & \Sigma_{15} & \Sigma_{16} & 0 & 0 & \Sigma_{19} & \Sigma_{110} \\
* & 0 & 0 & 0 & 0 & 0 & 0 & 0 & 0 & 0 \\
* & * & \Sigma_{33} & -Q_{2} & 0 & 0 & 0 & 0 & 0 & 0 \\
* & * & * & -Q_{3} & 0 & 0 & 0 & 0 & 0 & 0 \\
* & * & * & * & \Sigma_{55} & 0 & Z_{3} & 0 & 0 & 0 \\
* & * & * & * & * & \Sigma_{66} & Z_{4} & Z_{4} & 0 & 0 \\
* & * & * & * & * & * & \Sigma_{77} & 0 & 0 & 0 \\
* & * & * & * & * & * & * & -Q_{4}-Z_{4} & 0 & 0 \\
* & * & * & * & * & * & * & * & -\varepsilon I & 0 \\
* & * & * & * & * & * & * & * & * & -Z_{5}
\end{array}\right], \\
& \Sigma_{11}=c_{10} P(G \otimes D)+c_{10}(G \otimes D)^{T} P+Q_{1}+Q_{4}+Q_{6}+Q_{7}+r^{2} Z_{5}-\varepsilon \bar{U}-Z_{3}, \\
& \Sigma_{15}=\beta_{0} c_{20} P(G \otimes A)+Z_{3}, \quad \Sigma_{16}=\left(1-\beta_{0}\right) c_{20} P(G \otimes A), \quad \Sigma_{33}=-Q_{1}+Q_{3}, \\
& \Sigma_{19}=P-\varepsilon \bar{V}, \quad \Sigma_{110}=c_{30} P(G \otimes B), \quad \Sigma_{55}=-\left(1-\mu_{1}\right) Q_{5}-2 Z_{3}, \\
& \Sigma_{66}=-\left(1-\mu_{2}\right) Q_{6}-2 Z_{4}, \quad \Sigma_{77}=-Q_{3}+Q_{4}-Z_{3}-Z_{4} \text {, } \\
& \Gamma=\left[\sqrt{\beta_{0}} \Omega_{1}^{T} \quad \sqrt{1-\beta_{0}} \Omega_{2}^{T} \quad \delta_{1} \Omega_{3}^{T} \quad \sqrt{\beta_{0}} \delta_{2} \Omega_{4}^{T} \quad \sqrt{1-\beta_{0}} \delta_{2} \Omega_{5}^{T} \quad \delta_{3} \Omega_{6}^{T}\right], \\
& \Psi=\operatorname{diag}\{-Z,-Z,-Z,-Z,-Z,-Z\}, \\
& Z=\frac{1}{4} p^{2}\left(Z_{1}+Z_{2}\right)+h_{1}^{2} Z_{3}+\left(h_{2}-h_{1}\right)^{2} Z_{4} \\
& \Delta_{1}=\left[\begin{array}{llllllllll}
I & -I & 0 & 0 & 0 & 0 & 0 & 0 & 0 & 0
\end{array}\right] \text {, } \\
& \Delta_{2}=\left[\begin{array}{llllllllll}
0 & I & -I & 0 & 0 & 0 & 0 & 0 & 0 & 0
\end{array}\right] \text {, } \\
& \Delta_{3}=\left[\begin{array}{llllllllll}
0 & -I & I & 0 & 0 & 0 & 0 & 0 & 0 & 0
\end{array}\right] \text {, } \\
& \Delta_{4}=\left[\begin{array}{llllllllll}
0 & I & 0 & -I & 0 & 0 & 0 & 0 & 0 & 0
\end{array}\right] \text {, } \\
& \Delta_{5}=\left[\begin{array}{llllllllll}
I & 0 & 0 & 0 & -I & 0 & 0 & 0 & 0 & 0
\end{array}\right] \text {, } \\
& \Delta_{6}=\left[\begin{array}{llllllllll}
0 & 0 & 0 & 0 & I & 0 & -I & 0 & 0 & 0
\end{array}\right] \text {, } \\
& \Delta_{7}=\left[\begin{array}{llllllllll}
0 & 0 & 0 & 0 & 0 & -I & I & 0 & 0 & 0
\end{array}\right] \text {, } \\
& \Delta_{8}=\left[\begin{array}{llllllllll}
0 & 0 & 0 & 0 & 0 & I & 0 & -I & 0 & 0
\end{array}\right] \text {, } \\
& \Delta_{9}=\left[\begin{array}{llllllllll}
0 & 0 & I & I & 0 & 0 & 0 & 0 & 0 & 0
\end{array}\right] \text {, } \\
& \Delta_{10}=\left[\begin{array}{llllllllll}
I & 0 & I & 0 & 0 & 0 & 0 & 0 & 0 & 0
\end{array}\right] \text {, }
\end{aligned}
$$




$$
\begin{aligned}
& \Omega_{1}=\left[\begin{array}{lllllllllll}
c_{10} Z(G \otimes D) & Z K & 0 & 0 & c_{20} Z(G \otimes A) & 0 & 0 & 0 & Z & c_{30} Z(G \otimes B)
\end{array}\right], \\
& \Omega_{2}=\left[\begin{array}{lllllllllll}
c_{10} Z(G \otimes D) & Z K & 0 & 0 & 0 & c_{20} Z(G \otimes A) & 0 & 0 & Z & c_{30} Z(G \otimes B)
\end{array}\right],
\end{aligned}
$$

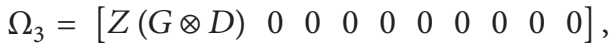

$$
\begin{aligned}
& \Omega_{4}=\left[\begin{array}{lllllllllll}
0 & 0 & 0 & 0 & Z(G \otimes A) & 0 & 0 & 0 & 0 & 0
\end{array}\right], \\
& \Omega_{5}=\left[\begin{array}{llllllllll}
0 & 0 & 0 & 0 & 0 & Z(G \otimes A) & 0 & 0 & 0 & 0
\end{array}\right], \\
& \Omega_{6}=\left[\begin{array}{llllllllll}
0 & 0 & 0 & 0 & 0 & 0 & 0 & 0 & 0 & Z(G \otimes B)
\end{array}\right] \text {. }
\end{aligned}
$$

Proof. Consider the following Lyapunov-Krasovskii (LK) functional:

$$
V(t)=V_{1}(t)+V_{2}(t)
$$

where

$$
\begin{aligned}
V_{1}(t)= & e^{T}(t) P e(t)+\int_{t-(1 / 2) p}^{t} \zeta^{T}(s)\left[\begin{array}{cc}
Q_{1} & Q_{2} \\
* & Q_{3}
\end{array}\right] \zeta(s) d s \\
& +\int_{t-h_{1}}^{t} e^{T}(s) Q_{4} e(s) d s+\int_{t-h_{2}}^{t-h_{1}} e^{T}(s) Q_{5} e(s) d s \\
& +\int_{t-\tau_{1}(t)}^{t} e^{T}(s) Q_{6} e(s) d s+\int_{t-\tau_{2}(t)}^{t} e^{T}(s) Q_{7} e(s) d s \\
V_{2}(t)= & \frac{1}{2} p \int_{-(1 / 2) p}^{0} \int_{t+\theta}^{t} \dot{e}^{T}(s) Z_{1} \dot{e}(s) d s d \theta \\
& +\frac{1}{2} p \int_{-p}^{-(1 / 2) p} \int_{t+\theta}^{t} \dot{e}^{T}(s) Z_{2} \dot{e}(s) d s d \theta \\
& +h_{1} \int_{-h_{1}}^{0} \int_{t+\theta}^{t} \dot{e}^{T}(s) Z_{3} \dot{e}(s) d s d \theta \\
& +\left(h_{2}-h_{1}\right) \int_{-h_{2}}^{-h_{1}} \int_{t+\theta}^{t} \dot{e}^{T}(s) Z_{4} \dot{e}(s) d s d \theta \\
& +r \int_{-r}^{0} \int_{t+\theta}^{t} e^{T}(s) Z_{5} e(s) d s d \theta \\
& \zeta(s)=c o l\left\{e(s), e\left(s-\frac{1}{2} p\right)\right\} .
\end{aligned}
$$

We use $L V(t)$ to denote the infinitesimal operator of $V(t)$, which is defined as

$$
L V(t)=\lim _{\Delta \rightarrow 0+} \Delta^{-1}[E\{V(t+\Delta) \mid e(t)\}-V(t)] .
$$

It follows from (19) and (21) that

$$
L V(t)=L V_{1}(t)+L V_{2}(t) .
$$

Now, taking the derivative of (22) along the solution of system (11) yields

$L V_{1}(t)$

$$
\begin{gathered}
\leq 2 e^{T}(t) P\left[\bar{g}(e(t))+c_{10}(G \otimes D) e(t)\right. \\
+\beta_{0} c_{20}(G \otimes A) e\left(t-\tau_{1}(t)\right) \\
+\left(1-\beta_{0}\right) c_{20}(G \otimes A) e\left(t-\tau_{2}(t)\right) \\
\left.\left.+c_{30}(G \otimes B)\right]_{t-r(t)}^{t} e(s) d s+K e(t-d(t))\right] \\
+\zeta^{T}(t)\left[\begin{array}{cc}
Q_{1} & Q_{2} \\
* & Q_{3}
\end{array}\right] \zeta(t) \\
-\zeta^{T}\left(t-\frac{1}{2} p\right)\left[\begin{array}{cc}
Q_{1} & Q_{2} \\
* & Q_{3}
\end{array}\right] \zeta\left(t-\frac{1}{2} p\right) \\
+e^{T}(t) Q_{4} e(t)-e^{T}\left(t-h_{1}\right) Q_{4} e\left(t-h_{1}\right) \\
+e^{T}\left(t-h_{1}\right) Q_{5} e\left(t-h_{1}\right) \\
-e^{T}\left(t-h_{2}\right) Q_{5} e\left(t-h_{2}\right)+e^{T}(t) Q_{6} e(t) \\
-\left(1-\mu_{1}\right) e^{T}\left(t-\tau_{1}(t)\right) Q_{6} e\left(t-\tau_{1}(t)\right)+e^{T}(t) Q_{7} e(t) \\
-\left(1-\mu_{2}\right) e^{T}\left(t-\tau_{2}(t)\right) Q_{7} e\left(t-\tau_{2}(t)\right)
\end{gathered}
$$

$L V_{2}(t)$

$$
\begin{aligned}
= & \dot{e}^{T}(t) Z \dot{e}(t)+r^{2} e^{T}(t) Z_{4} e(t) \\
& -\frac{1}{2} p \int_{t-(1 / 2) p}^{t} \dot{e}^{T}(s) Z_{1} \dot{e}(s) d s \\
& -\frac{1}{2} p \int_{t-p}^{t-(1 / 2) p} \dot{e}^{T}(s) Z_{2} \dot{e}(s) d s \\
& -h_{1} \int_{t-h_{1}}^{t} \dot{e}^{T}(s) Z_{3} \dot{e}(s) d s
\end{aligned}
$$




$$
\begin{aligned}
& -\left(h_{2}-h_{1}\right) \int_{t-h_{2}}^{t-h_{1}} \dot{e}^{T}(s) Z_{4} \dot{e}(s) d s \\
& -r \int_{t-r}^{t} e^{T}(s) Z_{5} e(s) d s .
\end{aligned}
$$

According to Lemma 7, we have

$$
\begin{aligned}
& -r \int_{t-r}^{t} e^{T}(s) Z_{5} e(s) d s \\
& \leq-r(t) \int_{t-r(t)}^{t} e^{T}(s) Z_{5} e(s) d s \\
& \quad \leq-\int_{t-r(t)}^{t} e^{T}(s) d s Z_{5} \int_{t-r(t)}^{t} e(s) d s .
\end{aligned}
$$

Denote $\eta_{1}(t)=\int_{t-d(t)}^{t} \dot{e}(s) d s, \eta_{2}(t)=\int_{t-(1 / 2) p}^{t-d(t)} \dot{e}(s) d s, \eta_{3}(t)=$ $\int_{t-d(t)}^{t-(1 / 2) p} \dot{e}(s) d s, \eta_{4}(t)=\int_{t-p}^{t-d(t)} \dot{e}(s) d s, v_{1}(t)=\int_{t-\tau_{1}(t)}^{t} \dot{e}(s) d s$, $v_{2}(t)=\int_{t-h_{1}}^{t-\tau_{1}(t)} \dot{e}(s) d s, v_{3}(t)=\int_{t-\tau_{2}(t)}^{t-h_{1}} \dot{e}(s) d s$, and $v_{4}(t)=$ $\int_{t-h_{2}}^{t-\tau_{2}(t)} \dot{e}(s) d s$. According to Lemma 8, if (16) holds, we have

$$
\begin{aligned}
& -h_{1} \int_{t-h_{1}}^{t} \dot{e}^{T}(s) U_{1} \dot{e}(s) d s \\
& \leq-\left[\begin{array}{l}
v_{1}(t) \\
v_{2}(t)
\end{array}\right]^{T}\left[\begin{array}{cc}
Z_{3} & S_{3} \\
* & Z_{3}
\end{array}\right]\left[\begin{array}{l}
v_{1}(t) \\
v_{2}(t)
\end{array}\right], \\
& -\left(h_{2}-h_{1}\right) \int_{t-h_{2}}^{t-h_{1}} \dot{e}^{T}(s) U_{2} \dot{e}(s) d s \\
& \leq-\left[\begin{array}{c}
v_{3}(t) \\
v_{4}(t)
\end{array}\right]^{T}\left[\begin{array}{cc}
Z_{4} & S_{4} \\
* & Z_{4}
\end{array}\right]\left[\begin{array}{l}
v_{3}(t) \\
v_{4}(t)
\end{array}\right] .
\end{aligned}
$$

Depending on whether the delay $d(t)$ belongs to the interval $0 \leq d(t) \leq(1 / 2) p$ or $(1 / 2) p \leq d(t) \leq p$, we will prove the result in two cases.

Case $1(0 \leq d(t) \leq(1 / 2) p)$. Using Lemmas 7 and 8 , one can obtain

$$
\begin{aligned}
& -\frac{1}{2} p \int_{t-(1 / 2) p}^{t} \dot{e}^{T}(s) Z_{1} \dot{e}(s) d s \\
& \quad \leq-\left[\begin{array}{l}
\eta_{1}(t) \\
\eta_{2}(t)
\end{array}\right]^{T}\left[\begin{array}{cc}
Z_{1} & S_{1} \\
* & Z_{1}
\end{array}\right]\left[\begin{array}{l}
\eta_{1}(t) \\
\eta_{2}(t)
\end{array}\right] \\
& -\frac{1}{2} p \int_{t-p}^{t-(1 / 2) p} \dot{e}^{T}(s) Z_{2} \dot{e}(s) d s \\
& \quad \leq-\left[\begin{array}{c}
e\left(t-\frac{1}{2} p\right) \\
\left.e\left(t-\frac{1}{2} p\right)\right]^{T}\left[\begin{array}{cc}
Z_{2} & Z_{2} \\
* & Z_{2}
\end{array}\right]\left[e\left(t-\frac{1}{2} p\right)\right.
\end{array}\right]
\end{aligned}
$$

Denote $\xi(t)=\operatorname{col}\{e(t), e(t-d(t)), e(t-(1 / 2) p), e(t-p), e(t-$ $\left.\left.\tau_{1}(t)\right), e\left(t-\tau_{2}(t)\right), e\left(t-h_{1}\right), e\left(t-h_{2}\right), \bar{g}(e(t)), \int_{t-r(t)}^{t} e(s) d s\right\}$,
$\Omega=\beta(t) \Omega_{10}+(1-\beta(t)) \Omega_{20}+\left(c_{1}(t)-c_{10}\right) \Omega_{30}+\beta(t)\left(c_{2}(t)-\right.$ $\left.c_{20}\right) \Omega_{40}+(1-\beta(t))\left(c_{2}(t)-c_{20}\right) \Omega_{50}+\left(c_{3}(t)-c_{30}\right) \Omega_{60}, \Omega_{10}=$

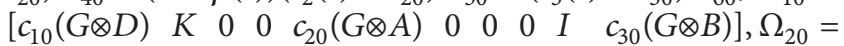

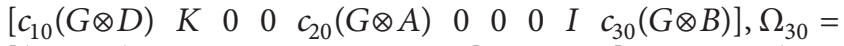

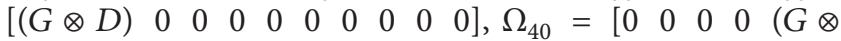

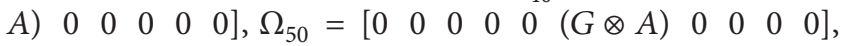
$\Omega_{60}=\left[\begin{array}{llllllllll}0 & 0 & 0 & 0 & 0 & 0 & 0 & 0 & 0 & (G \otimes B)\end{array}\right]$. Because $\beta(t)$ and $c_{k}(t)$ are mutually independent variables, it can be obtained that

$$
\begin{aligned}
E\left\{\dot{e}^{T}(t) Z \dot{e}(t)\right\} & =E\left\{\xi^{T}(t) \Omega^{T} Z \Omega \xi(t)\right\} \\
& =\xi^{T}(t) \bar{\Omega} \xi(t),
\end{aligned}
$$

where $\bar{\Omega}=\beta_{0} \Omega_{10}^{T} Z \Omega_{10}+\left(1-\beta_{0}\right) \Omega_{20}^{T} Z \Omega_{20}+\delta_{1}^{2} \Omega_{30}^{T} Z \Omega_{30}+$ $\beta_{0} \delta_{2}^{2} \Omega_{40}^{T} Z \Omega_{40}+\left(1-\beta_{0}\right) \delta_{2}^{2} \Omega_{50}^{T} Z \Omega_{50}+\delta_{3}^{2} \Omega_{60}^{T} Z \Omega_{60}$.

In addition, based on Assumption 5, for any scalar $\varepsilon>0$, we have

$$
y(t)=\varepsilon\left[\begin{array}{c}
e(t) \\
\bar{g}(e(t))
\end{array}\right]^{T}\left[\begin{array}{cc}
\bar{U} & \bar{V} \\
* & I
\end{array}\right]\left[\begin{array}{c}
e(t) \\
\bar{g}(e(t))
\end{array}\right] \leq 0 .
$$

Combining (23)-(29) and taking mathematical exceptions on both sides of (22) give

$$
E\{L V(t)\} \leq \xi^{T}(t)\left[\Phi_{1}+\bar{\Omega}\right] \xi(t) .
$$

Case $2((1 / 2) p \leq d(t) \leq p)$. Using Lemmas 7 and 8 , one can obtain

$$
\begin{aligned}
& -\frac{1}{2} p \int_{t-(1 / 2) p}^{t-(1 / 2) p} \dot{e}^{T}(s) Z_{2} \dot{e}(s) d s \\
& \quad \leq-\left[\begin{array}{l}
\eta_{3}(t) \\
\eta_{4}(t)
\end{array}\right]^{T}\left[\begin{array}{cc}
Z_{2} & S_{2} \\
* & Z_{2}
\end{array}\right]\left[\begin{array}{l}
\eta_{3}(t) \\
\eta_{4}(t)
\end{array}\right], \\
& -\frac{1}{2} p \int_{t-p}^{t} \dot{e}^{T}(s) Z_{1} \dot{e}(s) d s \\
& \quad \leq-\left[\begin{array}{c}
e(t) \\
e\left(t-\frac{1}{2} p\right)
\end{array}\right]^{T}\left[\begin{array}{cc}
Z_{1} & -Z_{1} \\
* & Z_{1}
\end{array}\right]\left[\begin{array}{c}
e(t) \\
e\left(t-\frac{1}{2} p\right)
\end{array}\right] .
\end{aligned}
$$

Similar to the above process, we also can obtain

$$
E\{L V(t)\} \leq \xi^{T}(t)\left[\Phi_{2}+\bar{\Omega}\right] \xi(t) .
$$

By the Schur complement, it is easy to derive that (17) is equivalent to $\Phi_{i}+\bar{\Omega}<0$. Let $\lambda=\min \left\{\lambda_{\min }\left(-\left(\Phi_{i}+\bar{\Omega}\right)\right)\right\}$. It follows from (30) and (32) that

$$
E\{L V(t)\} \leq-\lambda E\left\{\|\xi(t)\|^{2}\right\} \leq-\lambda E\left\{\|e(t)\|^{2}\right\} .
$$

Then, by the generalized Itô formula, we have

$$
\begin{aligned}
E\{V(t)\}-E\{V(0)\} & =E\left\{\int_{0}^{t} L V(s) d s\right\} \\
& \leq-\lambda \int_{0}^{t} E\left\{\|e(s)\|^{2}\right\} d s .
\end{aligned}
$$

Therefore, by virtue of (34), the discussion in [27], and Definition 6, system (11) is globally asymptotically stable in mean-square sense. This completes the proof. 
Remark 10. It is well known that delay decomposition method is helpful in the reduction of conservatism for the stability of delayed systems. In this paper, for the sake of obtaining some less conservative sufficient conditions and saving time consumed, a new LKF of form (19) is constructed, where the interval $[-p, 0]$ is divided into two divisions: $[-p,-(1 / 2) p]$ and $[-(1 / 2) p, 0]$. It is obvious that the $\operatorname{LKF}(19)$ can make good use of the information of additional error state $e(t-(1 / 2) p)$ sufficiently. Therefore, such an LKF is expected to be effective in the reduction of conservatism.

Remark 11. In the previous paper [21], by setting $\alpha=$ $d(t) / p$ and $\beta=\tau(t) / h$, based on Jensen's inequality technique, the integral terms $-p \int_{t-p}^{t} \dot{e}^{T}(s) Z_{1} \dot{e}(s) d s$ and $-h \int_{t-h}^{t} \dot{e}^{T}(s) Z_{2} \dot{e}(s) d s$ were upper-bounded by

$$
\begin{aligned}
& -p \int_{t-p}^{t} \dot{e}^{T}(s) Z_{1} \dot{e}(s) d s \\
& \leq-(2-\alpha)[e(t)-e(t-d(t))]^{T} \\
& \quad \times Z_{1}[e(t)-e(t-d(t))] \\
& -(1+\alpha)[e(t-d(t))-e(t-p)]^{T} \\
& \quad \times Z_{1}[e(t-d(t))-e(t-p)] \\
& -h \int_{t-h}^{t} \dot{e}^{T}(s) Z_{2} \dot{e}(s) d s \\
& \leq-(2-\beta)[e(t)-e(t-\tau(t))]^{T} \\
& \quad \times Z_{2}[e(t)-e(t-\tau(t))]
\end{aligned}
$$

$$
\begin{aligned}
& -(1+\beta)[e(t-\tau(t))-e(t-h)]^{T} \\
& \times Z_{2}[e(t-\tau(t))-e(t-h)],
\end{aligned}
$$

which result in a convex combination on $\alpha$ and $\beta$, respectively. As discussed in [26], reciprocally convex approach is an effective approach in handling the double integral terms of the LK functional for delayed systems, which can achieve performance behavior identical to the approaches based on the integral inequality lemma but with much less decision variables, comparable to those based on the Jensen inequality lemma. By utilizing the result of Lemma 8, Theorem 9 directly handles convex combination of quadratic terms of some integral terms, which leads to a less conservative result than [21].

In the following, we will explore how to design the desired sampled-data controllers to guarantee the complex network (6) synchronizing in mean square sense. Based on Theorem 9, we can easily derive the following theorem.

Theorem 12. Suppose that Assumptions 1, 2, 3, and 5 hold. The complex network (6) is globally asymptotically synchronized in mean square sense by the sampled-data controllers of form (10) if there exist matrices $P=\operatorname{diag}\left\{P_{1}, P_{2}, \ldots, P_{N}\right\}>0,\left[\begin{array}{cc}Q_{1} & Q_{2} \\ * & Q_{3}\end{array}\right]>$ $0, Q_{i}>0(i=4, \ldots, 7), Z_{i}>0(i=1, \ldots, 4), S_{i}(i=$ $1,2,3), X=\operatorname{diag}\left\{X_{1}, X_{2}, \ldots, X_{N}\right\}$ of appropriate dimensions and $a$ scalar $\varepsilon>0$ such that (16) and the following LMIs hold:

$$
\left[\begin{array}{ll}
\widehat{\Phi}_{i} & \widehat{\Gamma} \\
* & \widehat{\Psi}
\end{array}\right]<0, \quad i=1,2
$$

where

$$
\begin{aligned}
& \widehat{\Phi}_{1}=\widehat{\Sigma}-\Delta_{1}^{T} Z_{1} \Delta_{1}-\Delta_{2}^{T} Z_{1} \Delta_{2}-\Delta_{1}^{T} S_{1} \Delta_{2}-\Delta_{2}^{T} S_{1} \Delta_{1}-\Delta_{5}^{T} S_{3} \Delta_{6} \\
& -\Delta_{6}^{T} S_{3} \Delta_{5}-\Delta_{7}^{T} S_{4} \Delta_{8}-\Delta_{8}^{T} S_{4} \Delta_{7}-\Delta_{9}^{T} Z_{2} \Delta_{9} \\
& \widehat{\Phi}_{2}=\widehat{\Sigma}-\Delta_{3}^{T} Z_{2} \Delta_{3}-\Delta_{4}^{T} Z_{2} \Delta_{4}-\Delta_{3}^{T} S_{2} \Delta_{4}-\Delta_{4}^{T} S_{2} \Delta_{3}-\Delta_{5}^{T} S_{3} \Delta_{6}-\Delta_{6}^{T} S_{3} \Delta_{5}-\Delta_{7}^{T} S_{4} \Delta_{8}-\Delta_{8}^{T} S_{4} \Delta_{7}-\Delta_{10}^{T} Z_{1} \Delta_{10}, \\
& \Sigma=\left[\begin{array}{cccccccccc}
\Sigma_{11} & X & 0 & 0 & \Sigma_{15} & \Sigma_{16} & 0 & 0 & \Sigma_{19} & \Sigma_{110} \\
* & 0 & 0 & 0 & 0 & 0 & 0 & 0 & 0 & 0 \\
* & * & \Sigma_{22} & 0 & 0 & 0 & 0 & 0 & 0 & 0 \\
* & * & * & -Q_{2} & 0 & 0 & 0 & 0 & 0 & 0 \\
* & * & * & * & \Sigma_{55} & 0 & Z_{3} & 0 & 0 & 0 \\
* & * & * & * & * & \Sigma_{66} & Z_{4} & Z_{4} & 0 & 0 \\
* & * & * & * & * & * & \Sigma_{77} & 0 & 0 & 0 \\
* & * & * & * & * & * & * & -Q_{4}-Z_{4} & 0 & 0 \\
* & * & * & * & * & * & * & * & -\varepsilon I & 0 \\
* & * & * & * & * & * & * & * & * & -Z_{5}
\end{array}\right],
\end{aligned}
$$

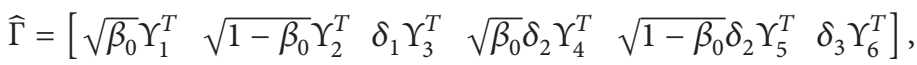

$$
\begin{aligned}
& \widehat{\Psi}=\operatorname{diag}\{-2 P+Z,-2 P+Z,-2 P+Z,-2 P+Z,-2 P+Z,-2 P+Z\},
\end{aligned}
$$




$$
\begin{aligned}
& \Upsilon_{1}=\left[\begin{array}{llllllllll}
c_{10} P(G \otimes D) & X & 0 & 0 & c_{20} P(G \otimes A) & 0 & 0 & 0 & P & c_{30} P(G \otimes B)
\end{array}\right], \\
& \Upsilon_{2}=\left[\begin{array}{lllllllllll}
c_{10} P(G \otimes D) & X & 0 & 0 & 0 & c_{20} P(G \otimes A) & 0 & 0 & P & c_{30} P(G \otimes B)
\end{array},\right.
\end{aligned}
$$

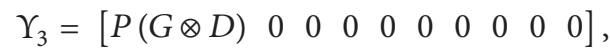

$$
\begin{aligned}
& \Upsilon_{4}=\left[\begin{array}{llllllllll}
0 & 0 & 0 & 0 & P(G \otimes A) & 0 & 0 & 0 & 0 & 0
\end{array}\right], \\
& \Upsilon_{5}=\left[\begin{array}{lllllllllll}
0 & 0 & 0 & 0 & 0 & P(G \otimes A) & 0 & 0 & 0 & 0
\end{array}\right], \\
& \Upsilon_{6}=\left[\begin{array}{llllllllll}
0 & 0 & 0 & 0 & 0 & 0 & 0 & 0 & 0 & P(G \otimes B)
\end{array}\right]
\end{aligned}
$$

and the other terms follow the same definitions as those in Theorem 9. Moreover, the desired controllers gain matrices are given by

$$
K_{i}=P_{i}^{-1} X_{i}, \quad i=1,2, \ldots, N
$$

Proof. Define matrices $J=\operatorname{diag}\left\{I, I, I, I, I, I, I, I, I, I, P Z^{-1}\right.$, $\left.P Z^{-1}, P Z^{-1}, P Z^{-1}, P Z^{-1}, P Z^{-1}\right\}$ and $X=P K$. Note that $-P Z^{-1} P \leq-2 P+Z$ is true for $Z>0$. Then, performing a congruence transformation of $J$ to (17), and considering the relation $X=P K$, we can obtain that if LMIs (36) is satisfied, then LMIs (17) holds. This completes the proof.

Remark 13. According to Theorem 12, though mixed probabilistic time-varying delays and random coupling strengths coexist in the considered complex network, the desired sampled-data controllers have been designed in terms of the solution to LMIs that can be solved effectively by using available software. In the next section, the effectiveness of the proposed method will be verified by some numerical examples.

\section{Numerical Examples}

In this section, two numerical examples are given to illustrate the effectiveness of the results proposed above.

Example 1. Consider complex network (6) with three nodes. We assume that the coupling strengths are some constants, time-varying coupling delay is deterministic, and the distributed coupling term vanishes. That is to say, $\beta(t)=1$, $c_{1}(t)=c_{10}, c_{2}(t)=c_{20}$, and $B=0$. The coupling configuration matrix is assumed to be

$$
G=\left[\begin{array}{ccc}
-1 & 0 & 1 \\
0 & -1 & 1 \\
1 & 1 & -2
\end{array}\right]
$$

The nonlinear function $f$ is taken as

$$
f\left(x_{i}(t)\right)=\left[\begin{array}{c}
-0.5 x_{i 1}+\tanh \left(0.2 x_{i 2}\right)+0.2 x_{i 2} \\
0.95 x_{i 2}-\tanh \left(0.75 x_{i 2}\right)
\end{array}\right] .
$$

TABLE 1: Maximum sampling interval $p$ for different $c_{20}$.

\begin{tabular}{lcc}
\hline Methods & 0.5 & 0.75 \\
\hline$[20]$ & 0.5409 & 0.1653 \\
{$[21]$} & 0.5573 & 0.2277 \\
Theorem 12 & 0.5891 & 0.2809 \\
\hline
\end{tabular}

It can be verified that $f$ satisfies (8) with

$$
U=\left[\begin{array}{cc}
-0.5 & 0.2 \\
0 & 0.95
\end{array}\right], \quad V=\left[\begin{array}{cc}
-0.3 & 0.2 \\
0 & 0.2
\end{array}\right]
$$

The time-varying coupling delay is chosen as $\tau(t)=0.2+$ $0.05 \sin (10 t)$. A straightforward calculation gives $h=0.25$ and $\mu=0.5$. The inner-coupling matrices are given as $D=0$ and $A=\left[\begin{array}{ll}1 & 0 \\ 0 & 1\end{array}\right]$. Thus, we only need to consider the effect of $c_{20}$. For different coupling strength $c_{20}$, Table 1 lists the maximum sampling interval $p$ obtained by Theorem 12 and $[20,21]$. From Table 1, we can see that our result is less conservative than the existing ones.

Example 2. The isolated node of the dynamical networks and the coupling configuration matrix $G$ are the same as Example 1. Let $c_{1}(t), c_{2}(t)$, and $c_{3}(t)$ are two mutually independent random variables satisfying normal distribution with $c_{10}=5, c_{20}=2, c_{20}=1, \delta_{1}=0.25, \delta_{2}=0.2$, and $\delta_{3}=0.1$. According to the property of normal distribution, almost all the values of $c_{k}(t), k=1,2,3$, satisfy $c_{i}(t) \in\left(c_{i 0}-\right.$ $\left.3 \delta_{i}, c_{i 0}+3 \delta_{i}\right)$; that is, $c_{1}(t) \in(4.25,5.75), c_{2}(t) \in(1.4,2.6)$, and $c_{3}(t) \in(0.7,1.3)$. Figures 1,2 , and 3 depict the random coupling strengths $c_{1}(t), c_{2}(t)$, and $c_{3}(t)$, respectively. Take $\beta_{0}=0.9, \tau_{1}(t)=0.2+0.1 \sin (t)$, and $\tau_{2}(t)=0.55+0.24 \sin (t)$. It followed that $h_{1}=0.3, h_{2}=0.79, \mu_{1}=0.1$, and $\mu_{2}=0.24$. Figure 4 shows the random coupling delay. The distributed coupling delay is chosen as $r(t)=0.5 \sin ^{2}(t)$, so we have $r=0.5$.

The inner-coupling matrices are given as

$$
D=A=\left[\begin{array}{cc}
0.1 & 0 \\
0 & 0.1
\end{array}\right], \quad B=\left[\begin{array}{cc}
0.4 & 0 \\
0 & 0.4
\end{array}\right]
$$




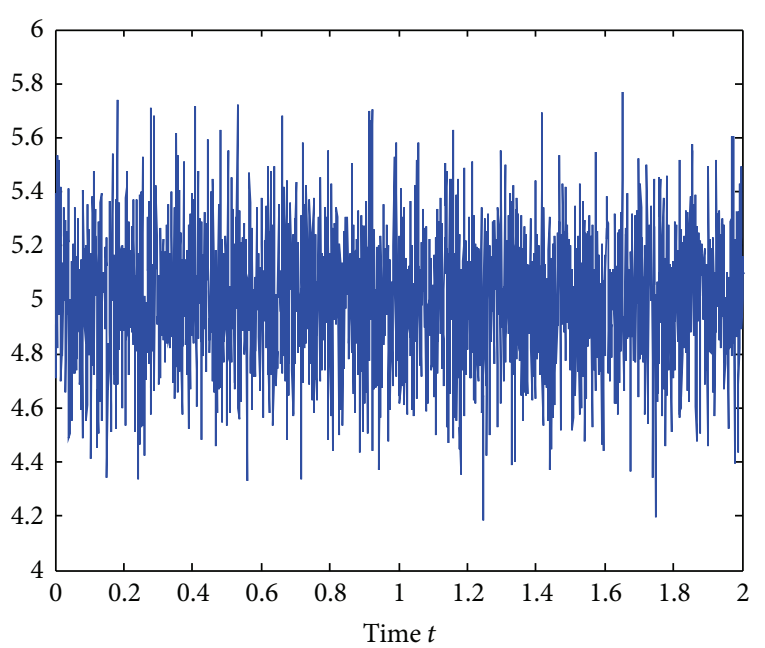

FIGURE 1: Random coupling strength $c_{1}(t)$.

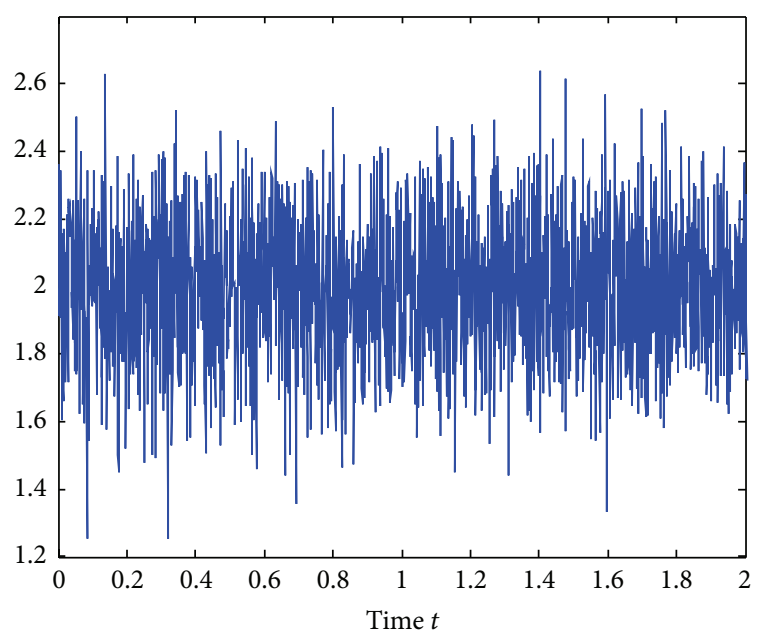

FIGURE 2: Random coupling strength $c_{2}(t)$.

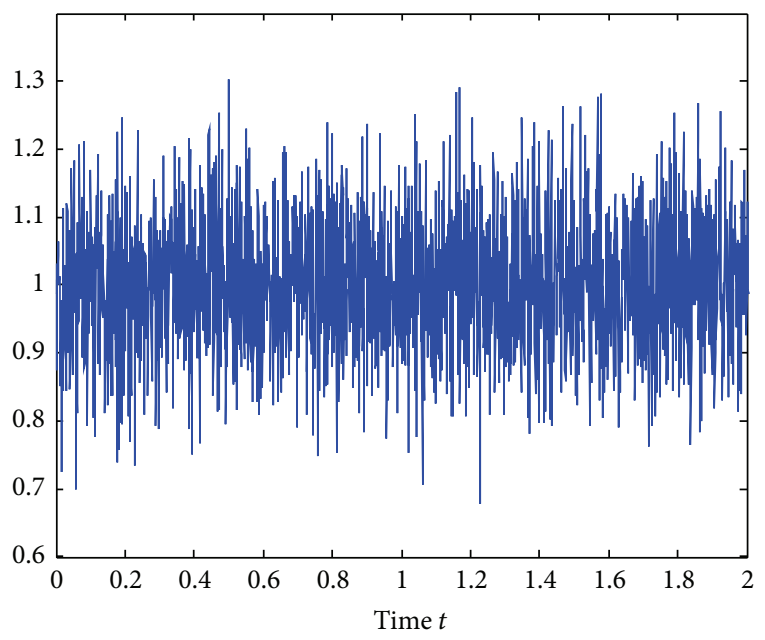

FIGURE 3: Random coupling strength $c_{3}(t)$.

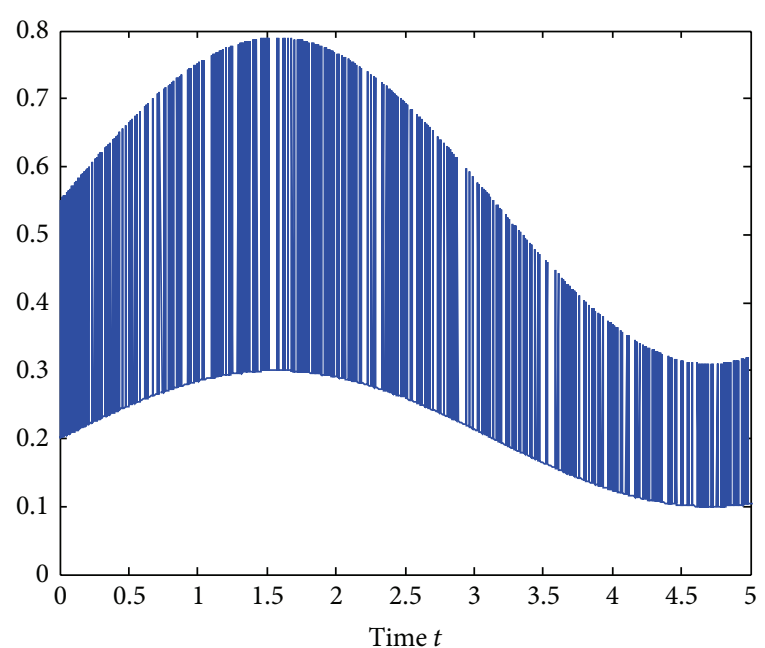

Figure 4: Random coupling delay $\tau(t)$.

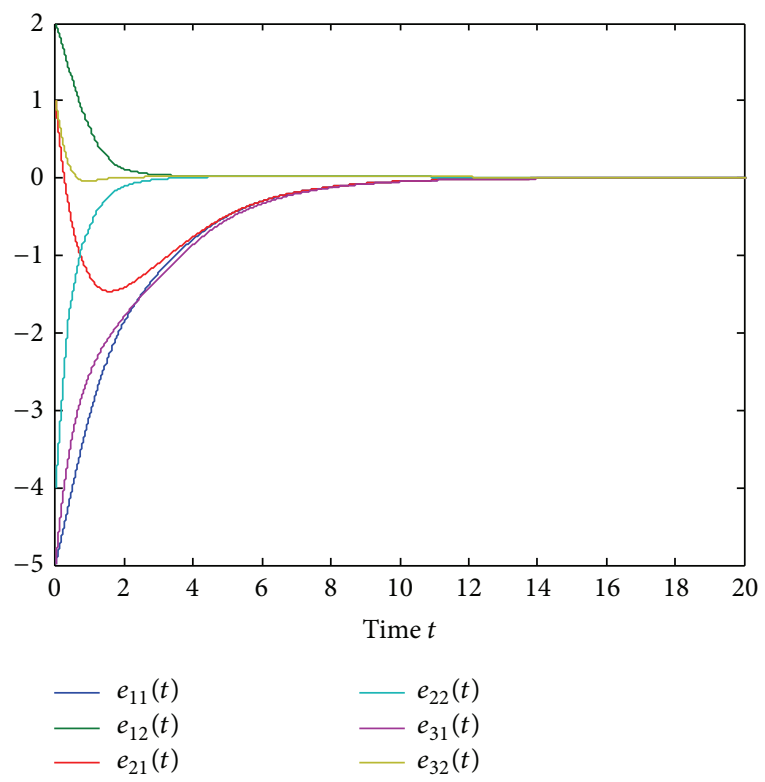

FIGURE 5: Synchronization error states.

Based on Theorem 12, the maximum value of sampling period is $p=0.4623$. Moreover, the gain matrices of the desired controllers can be obtained as follows:

$$
\begin{gathered}
K_{1}=\left[\begin{array}{ll}
-0.0647 & -0.1537 \\
-0.0048 & -1.1621
\end{array}\right], \quad K_{2}=\left[\begin{array}{ll}
-0.0647 & -0.1537 \\
-0.0048 & -1.1621
\end{array}\right], \\
K_{3}=\left[\begin{array}{cc}
0.1637 & -0.1603 \\
-0.0067 & -0.8554
\end{array}\right] .
\end{gathered}
$$

In the numerical simulation, the initial values are given by $x_{1}(0)=\left[\begin{array}{ll}-2 & 4\end{array}\right]^{T}, x_{2}(0)=\left[\begin{array}{ll}4 & -2\end{array}\right]^{T}, x_{3}(0)=\left[\begin{array}{ll}-2 & 3\end{array}\right]^{T}$, and $s(0)=\left[\begin{array}{ll}3 & 2\end{array}\right]^{T}$. The state trajectories of the synchronization error and the control inputs $u_{i}(t)$ are given in Figures 5 and 6 , 


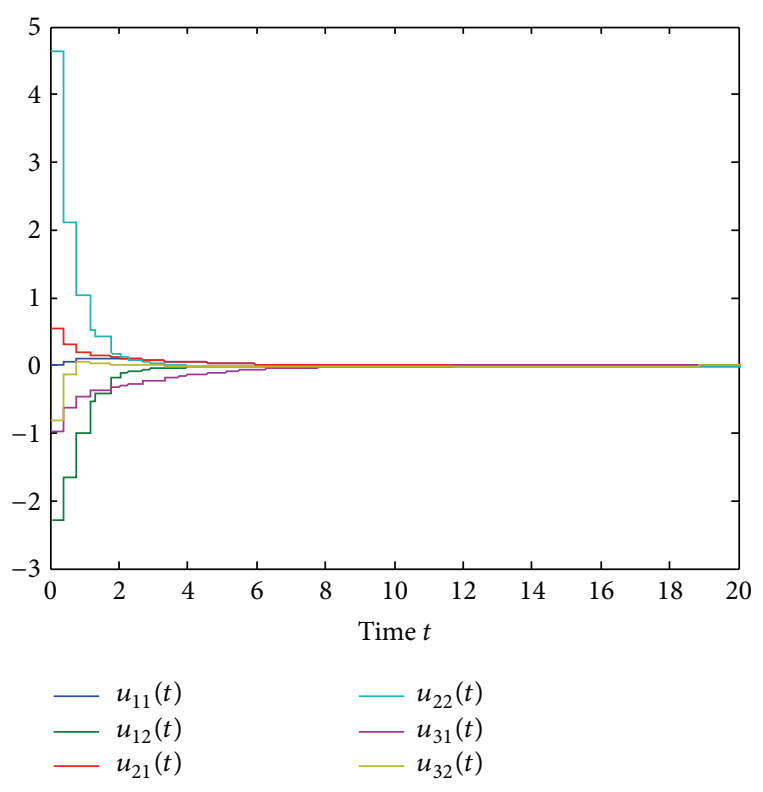

FIGURE 6: Sampled-data control inputs.

respectively. Clearly, the synchronization errors are globally asymptotically stable in mean square under the proposed sampled-data scheme.

\section{Conclusions}

In this paper, the sampled-data synchronization problem has been studied for complex networks with random coupling strengths and mixed probabilistic time-varying coupling delays. Based on random variables and the input delay approach, synchronization error dynamics are obtained. By using the delay decomposition method and reciprocally convex approach, a mean square synchronization criterion is derived, and the corresponding sampled-data controllers are designed in terms of the solution to LMIs. Numerical examples have shown the validity of the presented results. The LK functional considered here neglects the characteristic of sampled-data system, so it inevitably leads to some conservatism. Designing a less conservative sampled-data controller via taking the characteristic of sampled-data system into account constitutes a future research direction.

\section{Conflict of Interests}

The author declares that there is no conflict of interests regarding the publication of this paper.

\section{Acknowledgments}

The work is supported by the National Natural Science Foundation of China (Grants nos. 61203049 and 61303020), the Doctoral Startup Foundation of Taiyuan University of Science and Technology (Grant no. 20112010), and the Scientific and Technological Innovation Programs of Higher Education Institutions in Shanxi (Grant no. 20121068).

\section{References}

[1] S. H. Strogatz, "Exploring complex networks," Nature, vol. 410, no. 6825, pp. 268-276, 2001.

[2] M. E. J. Newman, "The structure and function of complex networks," SIAM Review, vol. 45, no. 2, pp. 167-256, 2003.

[3] J. H. Lü and G. R. Chen, "A time-varying complex dynamical network model and its controlled synchronization criteria," IEEE Transactions on Automatic Control, vol. 50, no. 6, pp. 841846, 2005.

[4] J. Q. Lu, D. W. C. Ho, and J. D. Cao, "A unified synchronization criterion for impulsive dynamical networks," Automatica, vol. 46, no. 7, pp. 1215-1221, 2010.

[5] Z. Li and J.-J. Lee, "New eigenvalue based approach to synchronization in asymmetrically coupled networks," Chaos, vol. 17, no. 4, Article ID 043117, 2007.

[6] Y.-W. Wang, J.-W. Xiao, C. Y. Wen, and Z.-H. Guan, "Synchronization of continuous dynamical networks with discrete-time communications," IEEE Transactions on Neural Networks, vol. 22, no. 12, pp. 1979-1986, 2011.

[7] X. Y. Guo and J. M. Li, "Stochastic adaptive synchronization for time-varying complex delayed dynamical networks with heterogeneous nodes," Applied Mathematics and Computation, vol. 222, pp. 381-390, 2013.

[8] P. Checco, M. Righero, M. Biey, and L. Kocarev, "Synchronization in networks of Hindmarsh-Rose neurons," IEEE Transactions on Circuits Systems II: Express Briefs, vol. 55, no. 12, pp. 1274-1278, 2008.

[9] J. W. Feng, Z. Tang, Y. Zhao, and C. Xu, "Cluster synchronisation of non-linearly coupled Lur'e networks with identical and nonidentical nodes and an asymmetrical coupling matrix," IET Control Theory \& Applications, vol. 7, no. 18, pp. 2117-2127, 2013.

[10] Y. Liang, X. Y. Wang, and J. Eustace, "Adaptive synchronization in complex networks with non-delay and variable delay couplings via pinning control," Neurocomputing, vol. 123, pp. 292298, 2014.

[11] J. Yu, C. Hu, H. J. Jiang, and Z. D. Teng, "Synchronization of nonlinear systems with delays via periodically nonlinear intermittent control," Communications in Nonlinear Science and Numerical Simulation, vol. 17, no. 7, pp. 2978-2989, 2012.

[12] X. S. Yang, S. Ai, T. T. Su, and A. C. Chang, "Synchronization of general complex networks with hybrid couplings and unknown perturbations," Abstract and Applied Analysis, vol. 2013, Article ID 625372, 14 pages, 2013.

[13] E. Fridman, A. Seuret, and J.-P. Richard, "Robust sampleddata stabilization of linear systems: an input delay approach," Automatica, vol. 40, no. 8, pp. 1441-1446, 2004.

[14] E. Fridman, "A refined input delay approach to sampled-data control," Automatica, vol. 46, no. 2, pp. 421-427, 2010.

[15] A. Seuret, "A novel stability analysis of linear systems under asynchronous samplings," Automatica, vol. 48, no. 1, pp. 177182, 2012.

[16] B. Shen, Z. D. Wang, and X. H. Liu, "A stochastic sampled-data approach to distributed $H_{\infty}$ filtering in sensor networks," IEEE Transactions on Circuits and Systems. I: Regular Papers, vol. 58, no. 9, pp. 2237-2246, 2011.

[17] J.-G. Lu and D. J. Hill, "Global asymptotical synchronization of chaotic Lur'e systems using sampled data: a linear matrix inequality approach," IEEE Transactions on Circuits and Systems II: Express Briefs, vol. 55, no. 6, pp. 586-590, 2008. 
[18] Z.-G. Wu, P. Shi, H. Y. Su, and J. Chu, "Exponential synchronization of neural networks with discrete and distributed delays under time-varying sampling," IEEE Transactions on Neural Networks and Learning Systems, vol. 23, pp. 1368-1376, 2012.

[19] C.-K. Zhang, L. Jiang, Y. He, Q. H. Wu, and M. Wu, "Asymptotical synchronization for chaotic Lur'e systems using sampleddata control," Communications in Nonlinear Science and Numerical Simulation, vol. 18, no. 10, pp. 2743-2751, 2013.

[20] N. Li, Y. L. Zhang, J. W. Hu, and Z. Y. Nie, "Synchronization for general complex dynamical networks with sampled-data," Neurocomputing, vol. 74, no. 5, pp. 805-811, 2011.

[21] Z.-G. Wu, J. H. Park, H. Y. Su, B. Song, and J. Chu, "Exponential synchronization for complex dynamical networks with sampled-data," Journal of the Franklin Institute, vol. 349, no. 9, pp. 2735-2749, 2012.

[22] Z.-G. Wu, P. Shi, H. Y. Su, and J. Chu, "Sampled-data exponential synchronization of complex dynamical networks with timevarying coupling delay," IEEE Transactions on Neural Networks and Learn Systems, vol. 24, no. 8, pp. 1177-1187, 2013.

[23] X. S. Yang, J. D. Cao, and J. Q. Lu, "Synchronization of coupled neural networks with random coupling strengths and mixed probabilistic time-varying delays," International Journal of Robust and Nonlinear Control, vol. 23, no. 18, pp. 2060-2081, 2013.

[24] X. S. Yang and J. D. Cao, "Synchronization of Markovian coupled neural networks with nonidentical mode-delays and random coupling strengths," IEEE Transactions on Neural Networks and Learn Systems, vol. 23, no. 1, pp. 60-71, 2012.

[25] H. Shao, "New delay-dependent stability criteria for systems with interval delay," Automatica, vol. 45, no. 3, pp. 744-749, 2009.

[26] P. Park, J. W. Ko, and C. Jeong, "Reciprocally convex approach to stability of systems with time-varying delays," Automatica, vol. 47, no. 1, pp. 235-238, 2011.

[27] L. Arnold, Stochastic Differential Equations: Theory and Applications, John Wiley \& Sons, New York, NY, USA, 1974. 


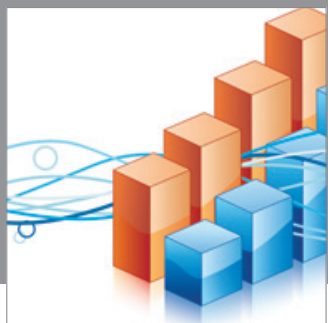

Advances in

Operations Research

mansans

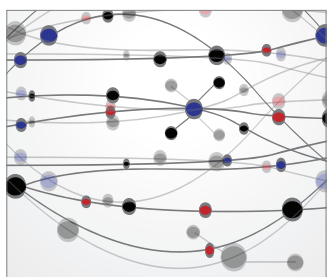

The Scientific World Journal
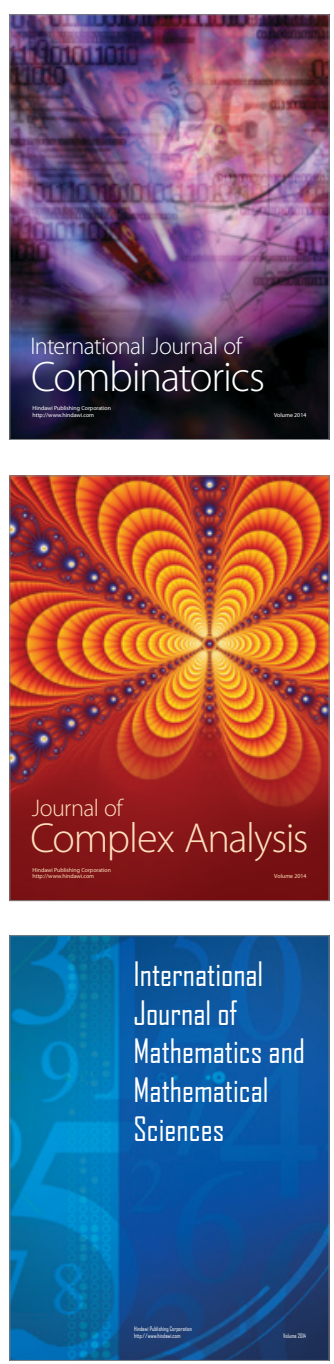
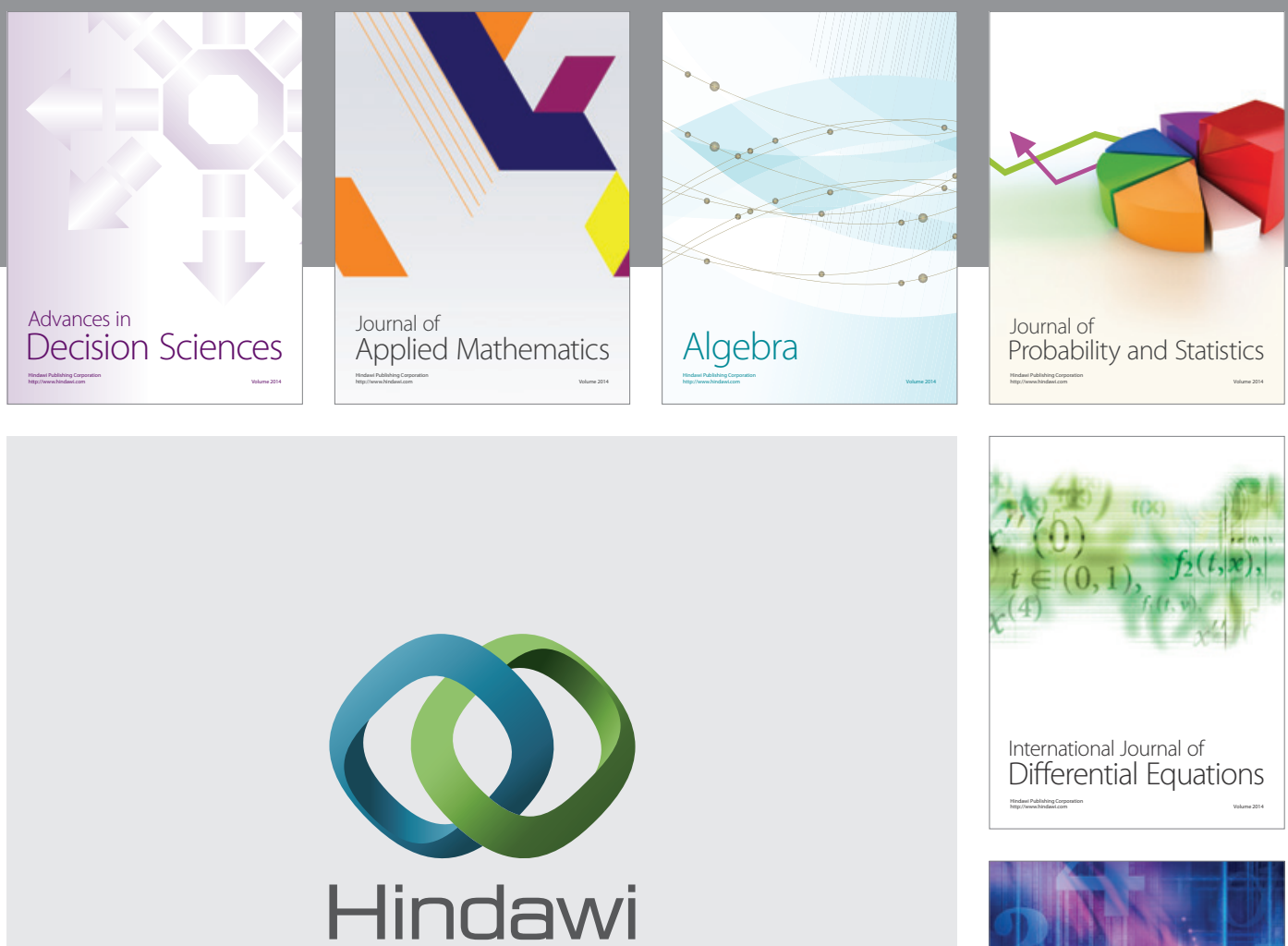

Submit your manuscripts at http://www.hindawi.com
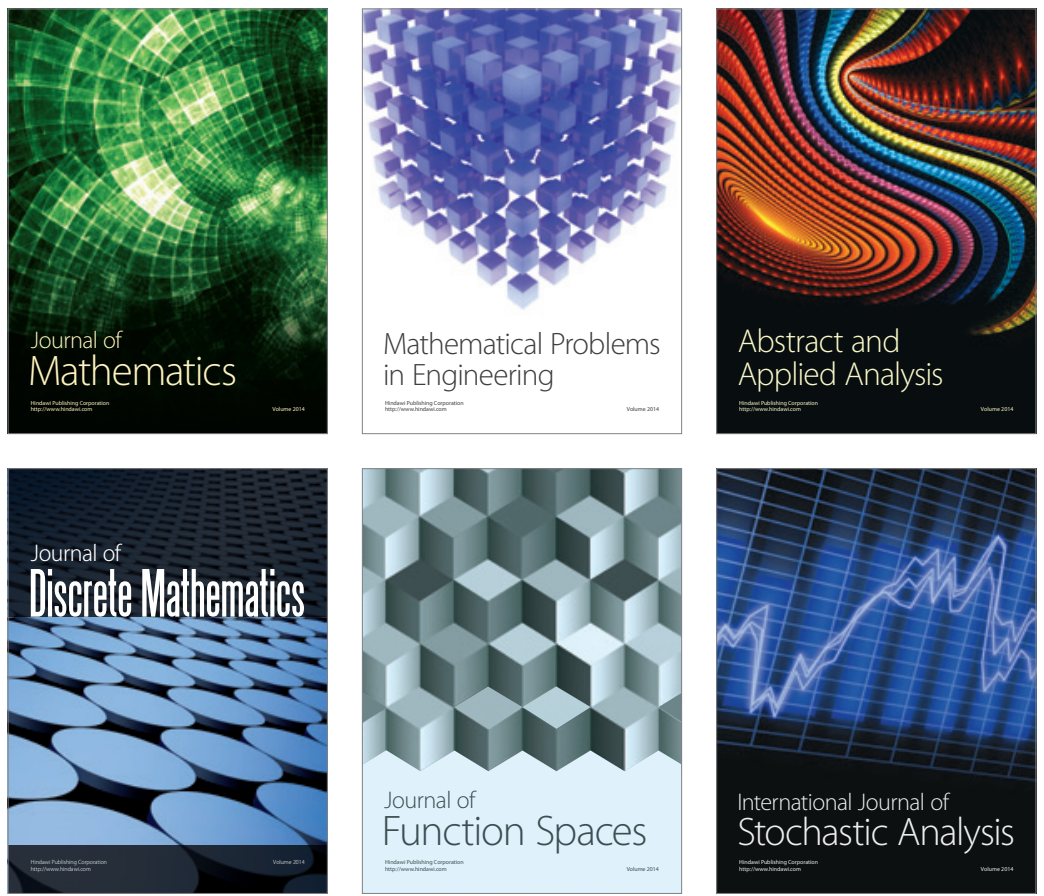

Journal of

Function Spaces

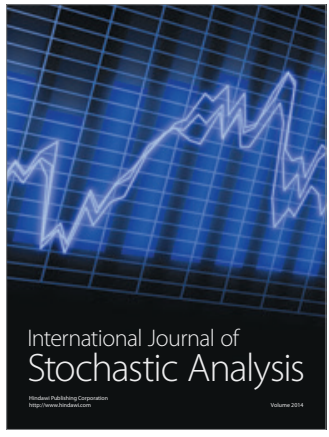

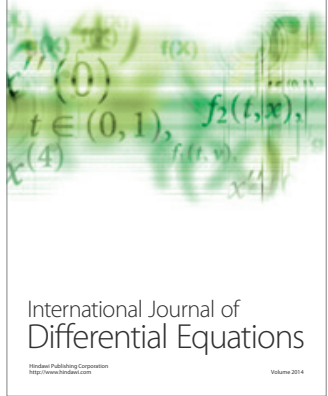
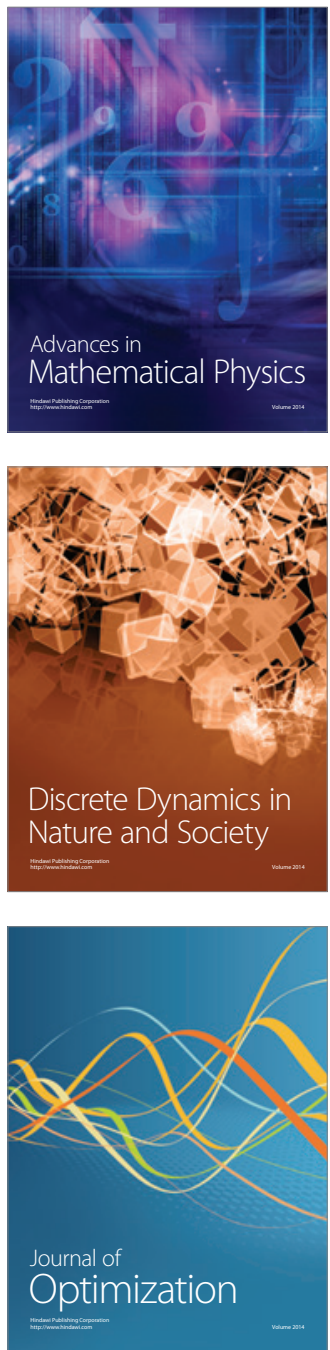